\title{
Additional Value of Preoperative Albumin for Surgical Risk Stratification among Colorectal Cancer Patients
}

\author{
David W. Larson ${ }^{a}$ Mohamed A. Abd El Aziz ${ }^{a}$ William Perry ${ }^{a}$ \\ Anne-Lise D'Angelo ${ }^{a}$ Kevin T. Behm ${ }^{a}$ Kellie L. Mathis ${ }^{a}$ Fabian Grass $^{b}$ \\ aDivision of Colon and Rectal Surgery, Department of Surgery, Mayo Clinic, Rochester, MN, USA; ${ }^{b}$ Department of \\ Visceral Surgery, Lausanne University Hospital CHUV, University of Lausanne, Lausanne, Switzerland
}

\section{Keywords}

European Society for Clinical Nutrition and Metabolism .

Malnutrition · Colorectal cancer · Surgery ·

Hypoalbuminemia

\begin{abstract}
Background: $\mathrm{BMI} \leq 18.5 \mathrm{~kg} / \mathrm{m}^{2}$ and preoperative weight loss may lead to inaccurate assessment of nutritional status, given the increasing prevalence of obesity. The aim of this study was to assess whether clinical evaluation of malnutrition based on these parameters is sufficient to predict complications after colorectal cancer surgery. Materials and Methods: The American College of Surgeons-National Quality Improvement Program database was queried from 2005 to 2018. Patients undergoing elective colorectal cancer surgery were divided into 4 groups: (1) albumin $<3.1 \mathrm{~g} / \mathrm{dL}$ within 21 days of surgery, (2) European Society for Clinical Nutrition and Metabolism (ESPEN) 2 clinical parameters for malnutrition ( $\geq 10 \%$ loss of weight $/ 6$ months plus $\left[\mathrm{BMI}<20 \mathrm{~kg} / \mathrm{m}^{2}\right.$ if age $<70$ years OR BMI $<22 \mathrm{~kg} / \mathrm{m}^{2}$ if age $\geq 70$ years]), (3) both aforementioned criteria, and (4) none of aforementioned criteria. Results: Of 82,280 patients, 5,932 (7.2\%) had hypoalbuminemia $<3.1 \mathrm{~g} / \mathrm{dL}, 764$ (0.9\%) fulfilled clinical ESPEN 2 parameters, and $338(0.4 \%)$ met both criteria. After adjusting for baseline confounders, patients in the hypoalbuminemia
\end{abstract}

karger@karger.com www.karger.com/anm

Karger $\%$

GOPEN ACCESS
(C) 2021 The Author(s)

Published by S. Karger AG, Basel

This is an Open Access article licensed under the Creative Commons Attribution-NonCommercial-4.0 International License (CC BY-NC) (http://www.karger.com/Services/OpenAccessLicense), applicable to the online version of the article only. Usage and distribution for commercial purposes requires written permission. group had a higher risk of overall complications (odds ratio [OR] $1.92, p<0.05$ vs. OR 1.18 in the ESPEN 2 group, $p<0.05$ ), major complications (OR 1.98, $p<0.05$ vs. OR 1.20, $p<0.05$ ), surgical complications (OR 1.77, 95\% $p<0.05$ vs. OR 1.1, $p>$ 0.05 ), medical complications (OR $1.73, p<0.05$ vs. OR 1.16 , $p>0.05$ ), surgical site infection (OR $1.32, p<0.05$ vs. OR 0.86 , $p>0.05$ ), and prolonged hospitalization (OR 1.79, $p<0.05$ vs. OR 1.22, $p<0.05)$. Patients who met both criteria were at highest risk. Conclusions: Preoperative measurement of serum albumin appears to be essential to identify patients at risk for complications after colorectal cancer surgery. Clinical evaluation through $\mathrm{BMI}$ and weight loss alone may underestimate surgery-associated risks in the USA.

(c) 2021 The Author(s)

Published by S. Karger AG, Basel

\section{Introduction}

Malnutrition is common in patients with colorectal cancer and associated with increased surgical morbidity, mortality, and compromised long-term outcomes [1-5] Preoperative nutritional screening is mandatory to identify patients who may benefit from nutritional therapy [6].

David W. Larson and Mohamed A. Abd El Aziz shared first authorship.

Fabian Grass

Department of Visceral Surgery, Lausanne University Hospital CHUV

University of Lausanne

Rue du Bugnon 46, CH-1011 Lausanne (Switzerland)

fabian.grass@chuv.ch 
However, defining universal diagnostic criteria for malnutrition independent of etiology and clinical setting represents a challenge [7]. A wide array of both clinical and laboratory parameters are available as nutritional screening tools [8-10]. However, there is no formal consensus regarding accuracy and value of different assessment methods in patients with colorectal cancer [11, 12], with differing guidelines between European and American nutritional societies $[6,10,13]$. Official screening tools as suggested by the European Society for Clinical Nutrition and Metabolism (ESPEN) integrate, besides measurements of body composition (muscle mass), low BMI and significant preoperative weight loss as mandatory clinical parameters $[10,14]$. Due to limited resources in a busy practice, measurement of body composition may not be routinely performed by many centers, leading to overly simplistic nutritional assessment through easily available clinical tools such as BMI and weight loss [15]. However, in countries with an increasing prevalence of obesity such as the USA, clinical evaluation based on BMI and weight loss alone may lead to an under-detection of patients at surgical risk $[6,16,17]$. In the absence of body composition as essential nutritional screening tool, serum albumin, often routinely assessed during preoperative screening, may help to refine surgical risk stratification [18].

Preoperative hypoalbuminemia of $<3.1 \mathrm{~g} / \mathrm{dL}$ has been recently identified as a critical threshold for postoperative adverse events [19]. However, systematic measurement of laboratory parameters such as serum albumin represents an additional cost burden to the chronically stressed health care system and a potential benefit remains questionable $[6,10,20]$. Since serum albumin does not represent a nutritional parameter but rather reflects disease severity, it was suggested as a helpful adjunct for risk stratification in ESPEN guidelines 2006 and the updated 2017 version $[20,21]$. The aim of this study was to evaluate a potential benefit of serum albumin as an adjunct to the clinical nutritional parameters BMI and weight loss in a representative US population undergoing colorectal cancer surgery.

\section{Methods}

\section{Data Source}

The American College of Surgeons-National Surgical Quality Improvement Program (ACS-NSQIP) Public User File from January 2005 to December 2018 provided the study cohort. ACS-NSQIP is an established, externally validated, and outcome-based clinical database that was created for quality improvement purposes. Trained data abstractors use standardized data extraction sheets to report demographic, anthropometric, and perioperative data from the participant sites, which represent a $20 \%$ random sample of the US surgical population.

\section{Cohort Selection}

All adult ( $\geq 18$ years) patients undergoing elective colorectal surgery for colon or rectal cancer using the Current Procedure Terminology codes and the International Classification of Diseases codes as specified in Figure 1 were included. Patients with missing anthropometric measures, missing albumin levels, albumin measurements deriving from outside the suggested preoperative 21-day window (half-life of albumin) [22], and American Society of Anesthesiologists (ASA) class V were excluded. Due to a potential impact on albumin levels, patients with preoperative ascites, preoperative sepsis, preoperative acute renal failure or hemodialysis, and ventilator dependence were excluded from the study.

Patients were divided into 4 non-overlapping, mutually exclusive groups: (1) severe hypoalbuminemia $<3.1 \mathrm{~g} / \mathrm{dL}$ [19], (2) clinical nutritional parameters for malnutrition $(\geq 10 \%$ weight loss over 6 months PLUS [BMI $<20 \mathrm{~kg} / \mathrm{m}^{2}$ in patients $<70$ years OR BMI $<22$ $\mathrm{kg} / \mathrm{m}^{2}$ in patients $\geq 70$ years] [10]), defined as ESPEN 2 parameters [7] (3) both aforementioned criteria, and (4) none of aforementioned criteria. For comparative purposes of the different groups, 2 additional definitions were used: ESPEN $1\left(\mathrm{BMI}<18.5 \mathrm{~kg} / \mathrm{m}^{2}\right)$ and moderate hypoalbuminemia (albumin $<3.5 \mathrm{~g} / \mathrm{dL}$ ). Of note, body composition (i.e., measurement of muscle mass) as a mandatory parameter for assessment of nutritional status according to ESPEN guidelines was not considered in this assessment. Therefore, the used clinical nutritional parameters should not be considered as officially recommended ESPEN screening tools.

ACS-NSQIP reported baseline demographics, laboratory parameters (albumin, hematocrit, platelet count, liver function tests i.e., serum glutamic oxaloacetic transaminase [SGOT]), and surgical details (approach, operating time, and extent of resection) were compared between the 4 groups [23]. Primary study outcomes of interest were postoperative 30-day complications according to standardized ACS-NSQIP definitions, which were regrouped as surgical (including surgical site infection [SSI], wound disruption, systemic sepsis, and need for blood transfusion), medical including urinary tract infection, respiratory complications (pneumonia, unplanned intubation, and/or on mechanical ventilator $\geq 48 \mathrm{~h}$ ), renal complications (progressive renal failure, and/or acute kidney injury), major adverse cardiovascular events (MACE: stroke, cardiac arrest requiring cardiopulmonary resuscitation, and/or myocardial infarction) and vascular thromboembolism (VTE: pulmonary embolism and/or deep venous thrombosis), minor complications (including urinary tract infection and superficial SSI), and major complications (including myocardial infarction, cardiac arrest requiring cardiopulmonary resuscitation, deep venous thrombosis, pulmonary embolism, pneumonia, unplanned intubation, need for mechanical ventilator for $\geq 48 \mathrm{~h}$ after surgery, acute kidney injury, progressive renal insufficiency, stroke, blood transfusion, deep SSI, organ space infection, wound disruption, and systemic sepsis). Further assessed were unplanned reoperation if the indication was related to the index operation, unplanned readmission related to the index operation, index length of stay (LOS, whereas prolonged LOS was defined as $\geq 7$ days according to the upper quartile of the entire cohort), and 30-day mortality. Postoperative complications were risk-adjusted and compared between the 3 groups (albumin $<3.1 \mathrm{~g} / \mathrm{dL}$, ESPEN 2, both criteria). 


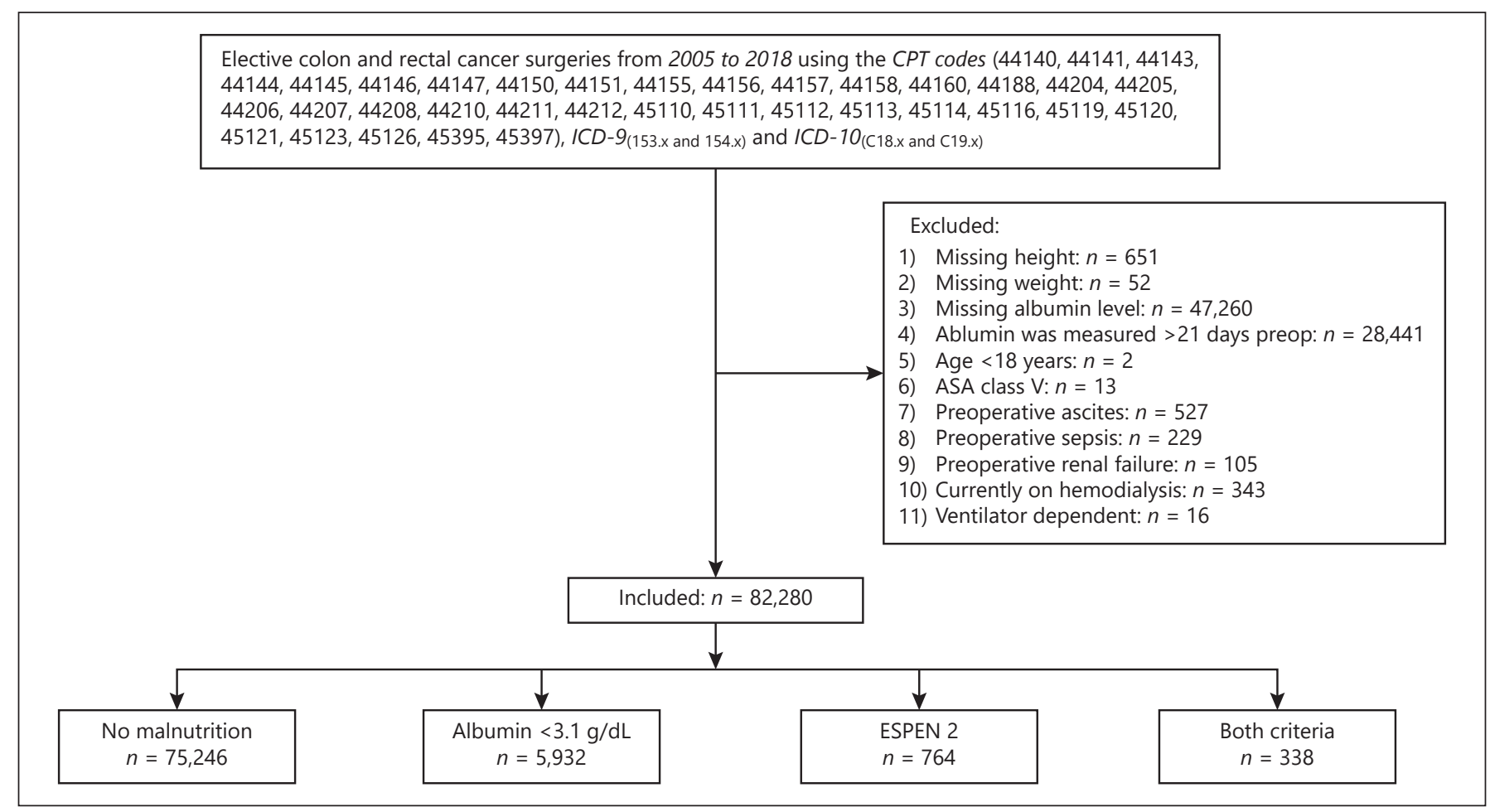

Fig. 1. Study's flow diagram. ESPEN, European Society for Clinical Nutrition and Metabolism.

\section{Statistical Analysis}

Descriptive statistics were reported as percentages and frequencies for categorical variables and as median (interquartile range) for continuous variables. The differences between exposure groups and outcomes were examined using the $\chi^{2}$ test for categorical variables and the Kruskal-Wallis test for continuous variables. Outcomes with an alpha level of less than 0.1 after univariable analysis were further evaluated by multivariable binary logistic regression to compute adjusted odds ratios (ORs). For all the analyses, an alpha level of less than 0.05 was considered statistically significant. Data analysis was performed using Statistical Package for the Social Sciences SPSS Advanced Statistics 25 (IBM Sofware Group, Inc., Armonk, NY, USA).

\section{Results}

A total of 82,280 patients constituted the study cohort. Of these, 5,932 (7.2\%) patients had preoperative hypoalbuminemia $<3.1 \mathrm{~g} / \mathrm{dL}, 764(0.9 \%)$ met the definition of ESPEN 2 clinical nutritional parameters, and 338 (0.4\%) met both criteria (hypoalbuminemia $<3.1 \mathrm{~g} / \mathrm{dL}$ and ESPEN 2), as detailed in Figure 1. Figure 2 illustrates the distribution of 3 different risk stratification methods ( $\geq 10 \%$ weight loss in the last 6 preoperative months, albumin $<3.5 \mathrm{~g} / \mathrm{dL}$, and BMI $<18.5 \mathrm{~kg} / \mathrm{m}^{2}$ ) among the $4 \mathrm{co}-$ horts according to suggested definitions (ESPEN 1, ESPEN 2 clinical nutritional parameters, severe hypoalbuminemia, and moderate hypoalbuminemia). Patients with severe hypoalbuminemia $<3.1 \mathrm{~g} / \mathrm{dL}$ were older, predominantly male, and had a higher rate of preoperative comorbidities, including diabetes mellitus, cardiac disorders, chronic steroid use, bleeding disorders, preoperative anemia, and high ASA class compared to patients who met clinical ESPEN 2 parameters (Table 1).

Patients with hypoalbuminemia $<3.1 \mathrm{~g} / \mathrm{dL}$ presented more overall complications ( 58.9 vs. $44.3 \%$ ), major complications (34.2 vs. $21.5 \%)$, surgical complications (31.6 vs. $20.3 \%)$, medical complications (17.4 vs. $10.2 \%)$, unplanned readmission (11.1 vs. 7.9\%), unplanned reoperation (5.4 vs. $4.6 \%$ ), prolonged LOS ( 40.3 vs. $29.6 \%$ ), and 30 -day mortality (5.2 vs. $2.2 \%$ ) than patients in the ESPEN 2 group (all $p<0.05$ ). Patients who met both criteria (albumin $<3.1 \mathrm{~g} / \mathrm{dL}$ and ESPEN 2) presented consistently the highest complication rates, as detailed in Table 2.

After adjusting for baseline confounders (using the baselined cohort not meeting any of the 3 suggested criteria as a reference), patients in the hypoalbuminemia group had a higher adjusted odds of overall complications (OR 1.92, 95\% CI [1.80-2.04] vs. OR 1.184, 95\% CI 


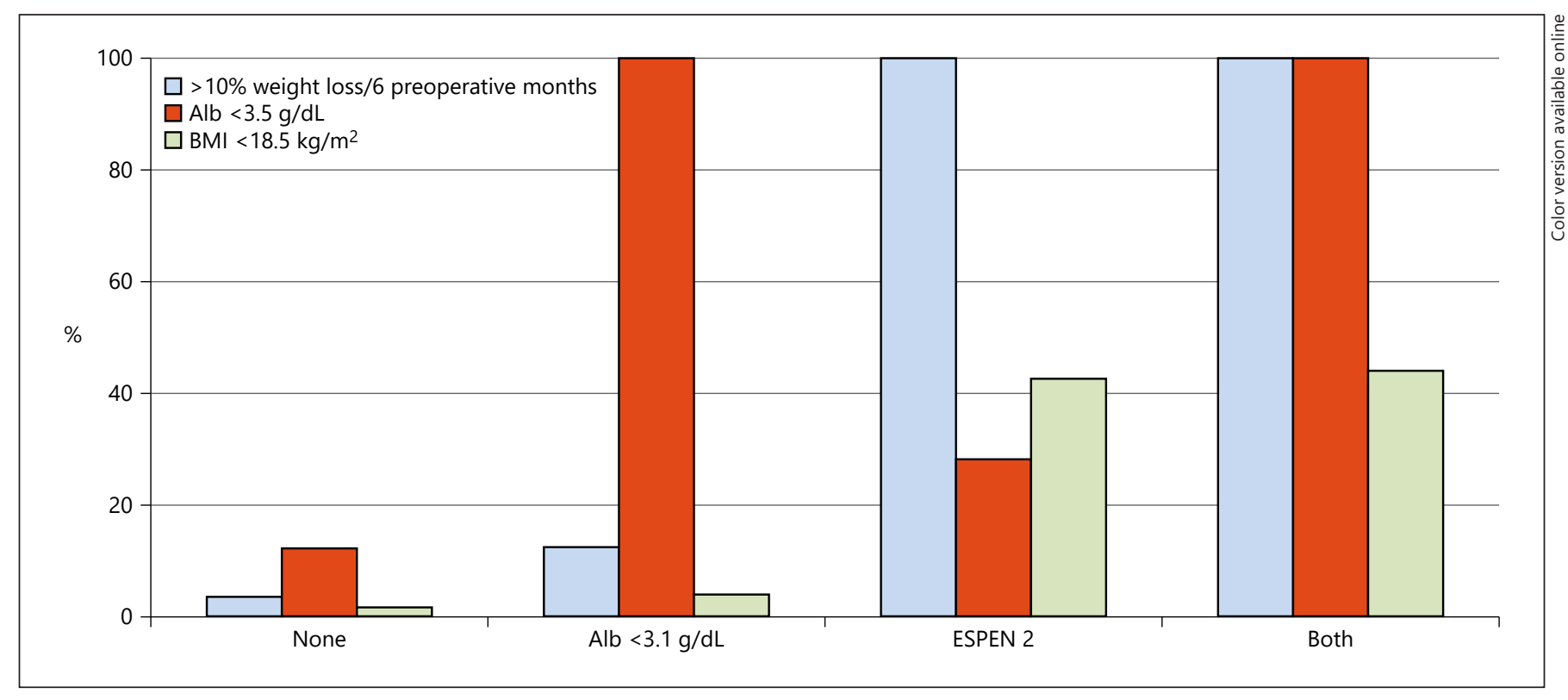

Fig. 2. Prevalence of different criteria of assessment among the study cohorts. ESPEN 2 defined as $\geq 10 \%$ weight $/ 6$ months plus $\left[\mathrm{BMI}<20 \mathrm{~kg} / \mathrm{m}^{2}\right.$ in patients $<70$ years OR BMI $<22 \mathrm{~kg} / \mathrm{m}^{2}$ in patients $\geq 70$ years]). ESPEN, European Society for Clinical Nutrition and Metabolism.

[1.01-1.39] in the ESPEN 2 group), major complications (OR 1.98, 95\% CI [1.86-2.11] vs. OR 1.20, 95\% CI [1.001.45]), surgical complications (OR 1.77, 95\% CI [1.66$1.88]$ vs. OR $1.1,95 \% \mathrm{CI}$ [0.90-1.33]), medical complications (OR 1.73, 95\% CI [1.59-1.87] vs. OR 1.16, 95\% CI [0.90-1.48]), SSI (OR 1.32, 95\% CI [1.21-1.44] vs. OR $0.86,95 \%$ CI [0.66-1.12]), prolonged hospitalization (OR $1.79,95 \%$ CI [1.68-1.91] vs. OR $1.22,95 \%$ CI [1.03-1.46]) compared to patients in the ESPEN 2 group (Fig. 3 and see online suppl. Table 1; for all online suppl. material, see www.karger.com/doi/10.1159/000514058). Patients who met both criteria (severe hypoalbuminemia and clinical ESPEN 2 parameters) were at highest risk of overall complications (OR 2.6, 95\% CI [2.03-3.33]), major complications (OR 2.51, 95\% CI [1.98-3.18]), and surgical complications (OR 2.26, 95\% CI [1.79-2.87]) (Fig. 3; online suppl. Table 1).

\section{Discussion}

This large-scale analysis derived from a national, validated data registry of elective surgical colorectal cancer patients revealed important differences in risk stratification depending on the screening modality. While less than $1 \%$ of this representative US cohort presented with malnutrition according to the suggested clinical nutritional parameters (BMI, weight loss), $7.2 \%$ of patients were at surgical risk according to the previously identified critical albumin threshold of $3.1 \mathrm{~g} / \mathrm{dL}$. Given the significant association of hypoalbuminemia and postoperative morbi-mortality, preoperative albumin levels may represent a critical adjunct to clinical screening tools to prevent under-detection of surgical risk patients. This is important, given the challenges to adopt the full panel of nutritional screening tools as recommended by ESPEN in an everyday practice setting [15].

Clinical tools integrating BMI may underestimate the presence of malnutrition, especially in the context of an increasing prevalence of obesity in the USA [17]. This was also acknowledged by the respective European consensus statements $[10,24]$. BMI has been criticized as an inaccurate measure of body fat content as it does not take into account muscle mass, bone density, overall body composition including fluid homeostasis, and racial and sex differences [25]. Furthermore, patients with obesity presenting with significant preoperative cachexia may remain undetected despite the important impact on biochemical, metabolic, and anthropometric body composition, while BMI scores remain within normal limits [16]. In this present study, only $4 \%$ of patients with severe preoperative hypoalbuminemia $<3.1 \mathrm{~g} / \mathrm{dL}$ had a $\mathrm{BMI}<18.5 \mathrm{~kg} / \mathrm{m}^{2}($ ES- 
Table 1. Baseline demographic, anthropometric, and preoperative characteristics

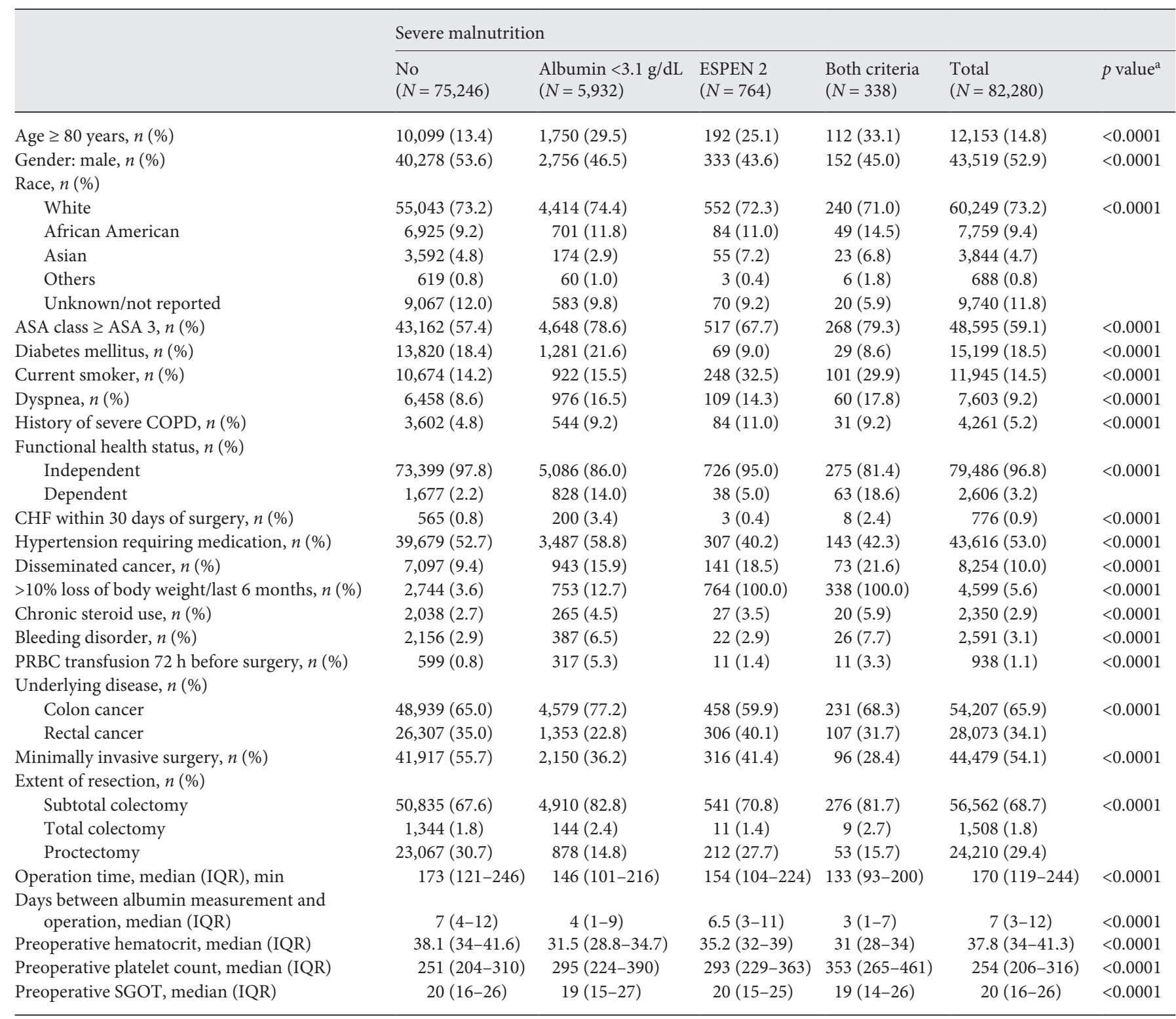

ESPEN 2: $\geq 10 \%$ weight $/ 6$ months plus (BMI $<20 \mathrm{~kg} / \mathrm{m}^{2}$ in patients $<70$ years OR BMI $<22 \mathrm{~kg} / \mathrm{m}^{2}$ in patients $\geq 70$ years) (body composite was not considered for our analysis). ASA, American Society of Anesthesiologists; COPD, chronic obstructive pulmonary disease; PRBC, packed red blood cells; IQR, interquartile range; ESPEN, European Society for Clinical Nutrition and Metabolism; SGOT, serum glutamic oxaloacetic transaminase. ${ }^{\mathrm{a}}$ Derived from the $\chi^{2}$ test for categorical variables and Independent sample Kruskal-Wallis test for continuous variables.

PEN 1 definition), while $13 \%$ lost $\geq 10 \%$ of their body weight (Fig. 2). This raises the question of insufficient performance of simplified clinical nutritional assessment in the US population. This is supported by the more significant correlation of hypoalbuminemia and risk-adjusted postoperative complications (Fig. 3).

While the expert panel reached a consensus regarding clinical criteria for malnutrition, systematic biochemical assessment was considered but ultimately excluded from the recommendation [10]. Additional costs with a questionable additional benefit in the decision-making process may have been prevailing arguments [26], despite the proven association of hypoalbuminemia and worse shortterm outcomes [19,27-29]. The critical cutoff value of 3.1 $\mathrm{g} / \mathrm{dL}$ has recently been identified by Haskins et al. [19], yielding $80 \%$ specificity for adverse events in patients 
Table 2. Postoperative complications

\begin{tabular}{|c|c|c|c|c|c|c|}
\hline & \multicolumn{6}{|c|}{ Severe malnutrition } \\
\hline Overall complications, ${ }^{*} n(\%)$ & $26,780(35.6)$ & $3,481(58.9)$ & $338(44.3)$ & $227(67.6)$ & $30,826(37.5)$ & $<0.0001$ \\
\hline Minor complications, $n(\%)$ & $5,516(7.3)$ & $634(10.7)$ & $53(6.9)$ & $38(11.2)$ & $6,241(7.6)$ & $<0.0001$ \\
\hline Any SSI & $7,097(9.4)$ & $780(13.1)$ & $67(8.8)$ & $45(13.3)$ & $7,989(9.7)$ & $<0.0001$ \\
\hline Superficial incisional & $3,749(5.0)$ & $397(6.7)$ & $28(3.7)$ & $25(7.4)$ & $4,199(5.1)$ & $<0.0001$ \\
\hline Deep incisional & $767(1.0)$ & $100(1.7)$ & $4(0.5)$ & $4(1.2)$ & $875(1.1)$ & $<0.0001$ \\
\hline Organ/space & $2,895(3.8)$ & $319(5.4)$ & $38(5.0)$ & $18(5.3)$ & $3,270(4.0)$ & $<0.0001$ \\
\hline Wound disruption & $704(0.9)$ & $91(1.5)$ & $13(1.7)$ & $6(1.8)$ & $814(1.0)$ & $<0.0001$ \\
\hline Systemic sepsis & $2,813(3.7)$ & $503(8.5)$ & $36(4.7)$ & $33(9.8)$ & $3,385(4.1)$ & $<0.0001$ \\
\hline UTI & $1,983(2.6)$ & $278(4.7)$ & $27(3.5)$ & $18(5.3)$ & $2,306(2.8)$ & $<0.0001$ \\
\hline Respiratory complication & $2,183(2.9)$ & $524(8.8)$ & $39(5.1)$ & $36(10.7)$ & $2,782(3.4)$ & $<0.0001$ \\
\hline Renal complication & $930(1.2)$ & $145(2.4)$ & $3(0.4)$ & $6(1.8)$ & $1,084(1.3)$ & $<0.0001$ \\
\hline MACE & $866(1.2)$ & $181(3.1)$ & $15(2.0)$ & $13(3.8)$ & $1,075(1.3)$ & $<0.0001$ \\
\hline VTE & $1,044(1.4)$ & $200(3.4)$ & $8(1.0)$ & $11(3.3)$ & $1,263(1.5)$ & $<0.0001$ \\
\hline LOS, median (IQR), days & $5(3-7)$ & $7(5-10)$ & $6(4-8)$ & $7(5-11)$ & $5(4-7)$ & $<0.0001$ \\
\hline LOS $>7$ days (Q3), $n(\%)$ & $16,385(21.8)$ & $2,382(40.3)$ & $226(29.6)$ & $150(44.6)$ & $19,143(23.3)$ & $<0.0001$ \\
\hline $\begin{array}{l}\text { Unplanned readmission related to the } \\
\text { primary procedure, }{ }^{*} n(\%)\end{array}$ & $4,723(8.8)$ & $357(11.1)$ & $38(7.9)$ & $22(13.4)$ & $5,140(8.9)$ & $<0.0001$ \\
\hline Unplanned reoperation related to the & & & & & & \\
\hline primary procedure, ${ }^{\#} n(\%)$ & $2,140(4.0)$ & $173(5.4)$ & $22(4.6)$ & $6(3.7)$ & $2,341(4.0)$ & $<0.0001$ \\
\hline
\end{tabular}

ESPEN 2: $\geq 10 \%$ weight $/ 6$ months plus (BMI $<20 \mathrm{~kg} / \mathrm{m}^{2}$ in patients $<70$ years OR BMI $<22 \mathrm{~kg} / \mathrm{m}^{2}$ in patients $\geq 70$ years) $($ body composite was not considered for our analysis). ESPEN, European Society for Clinical Nutrition and Metabolism; SSI, surgical site infection; UTI, urinary tract infection; MACE, major adverse cardiovascular events; VTE, vascular thromboembolism; LOS, length of stay. ${ }^{*}$ Overall complications include any surgical complication, any medical complication, LOS $>7$ days, unplanned readmission related to the primary operation, unplanned reoperation related to the primary operation, and 30-day mortality. ${ }^{*}$ Analyzed from 2012 to 2018 only (not captured before 2012). a Derived from $\chi^{2}$ test for categorical variables and independent-sample Kruskal-Wallis test for continuous variables.

with colon cancer. Our analysis revealed that only $0.9 \%$ of the entire cohort met the suggested cutoffs for clinical nutritional parameters (BMI and weight loss) without concomitant severe hypoalbuminemia (albumin $<3.1 \mathrm{~g} /$ dL, Fig. 1). Among all patients meeting our suggested clinical "ESPEN 2" parameters, only $31 \%$ presented with albumin $<3.1 \mathrm{~g} / \mathrm{dL}$. On the other hand, $95 \%$ (7.2\% of the entire cohort) of patients with albumin $<3.1 \mathrm{~g} / \mathrm{dL}$ did not meet the suggested clinical nutritional parameters. Taken together, a significant proportion of patients at risk may remain undetected without additional assessment of albumin levels. However, it is important to mention that nutritional assessment in the present study did not include measurement of body composition, as recommended by ESPEN guidelines.

The additional use of serum albumin as an adjunct to clinical assessment has been recommended by ESPEN guidelines [10, 20, 21, 30]. In addition, it was also recommended by the American Society of Enhanced Recovery and Perioperative Quality [6]. Nevertheless, albumin does not represent a nutritional marker per se but rather an additional tool for preoperative risk stratification.

Taken together, our findings suggest that clinical nutritional parameters (BMI and weight loss) may be insuf- 


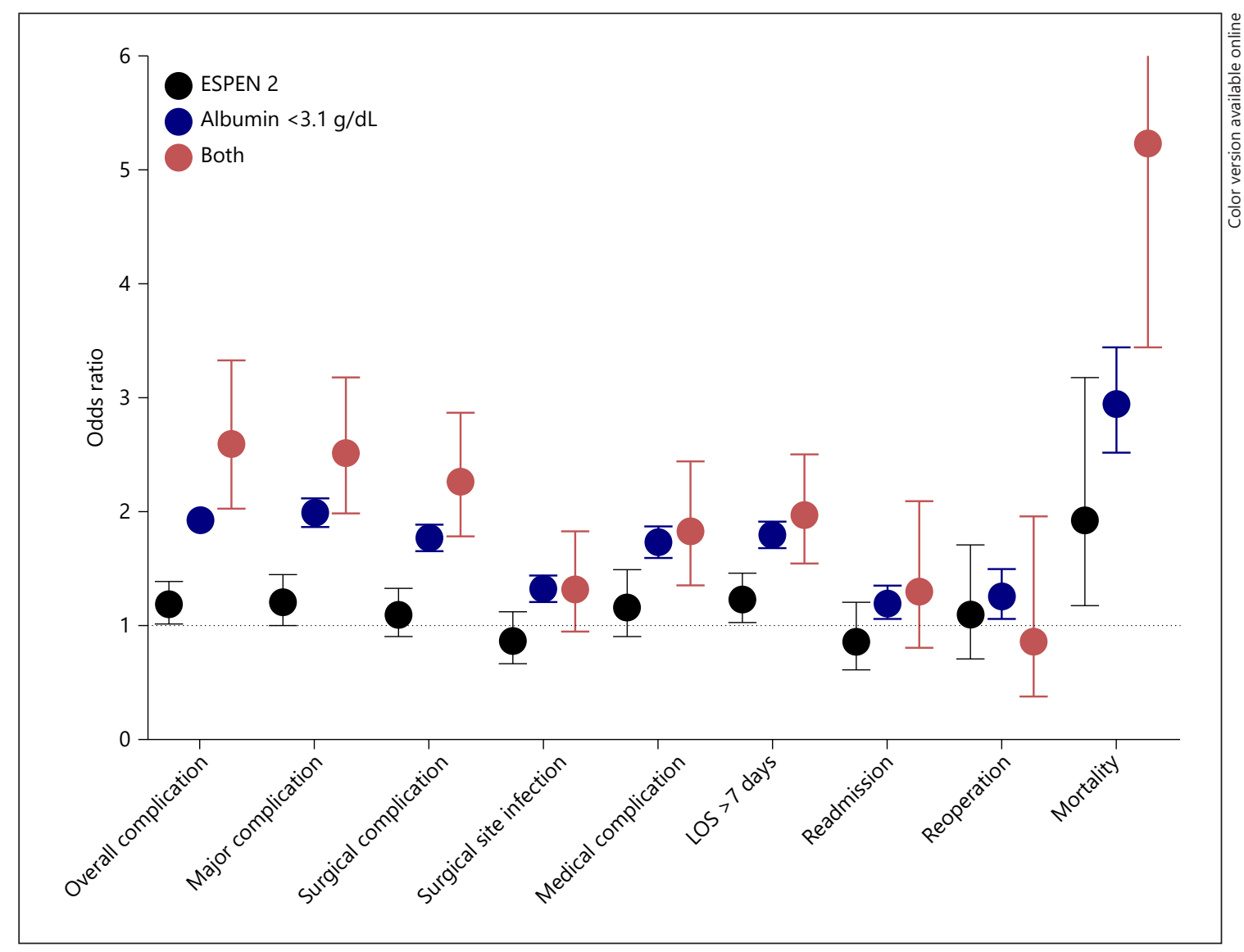

Fig. 3. Risk-adjusted comparison of postoperative outcomes. Comparison of postoperative outcomes between the 3 groups albumin $<3.1 \mathrm{~g} / \mathrm{dL}$, ESPEN 2, and both. Displayed are OR and $95 \%$ confidence interval. ESPEN 2: $\geq 10 \%$ weight $/ 6$ months plus (BMI $<20 \mathrm{~kg} / \mathrm{m}^{2}$ in patients $<70$ years OR BMI $<22 \mathrm{~kg} / \mathrm{m}^{2}$ in patients $\geq 70$ years) (body composite was not considered for our analysis). None of the 3 criteria met was used as a reference. Adjusted for age $>80$ years, gender, race, ASA class $\geq 3$, diabetes mellitus, current smoker within 1 year of surgery, dyspnea, history of severe COPD, functional status, hypertension requiring medication, disseminated

ficient for preoperative risk stratification in the US population with colorectal cancer. Validated nutritional screening tools (i.e., Nutritional Risk Score NRS-2002 [14], Malnutrition Universal Screening Tool MUST [31]), measurement of body composition (i.e., muscle mass) and close collaboration with nutritional specialists remain mandatory to tailor individual nutritional support strategies [17, 32]. Albumin measurement may thus be critical to refine indications for preoperative nutritional conditioning [33-35]. This strategy is in line with the preoperative nutrition screening (PONS score) algorithm, which has been proposed by The American Society for Enhanced Recovery and Perioperative Quality cancer, steroid use for chronic condition, bleeding disorders, blood transfusion $\geq 1$ units within $72 \mathrm{~h}$ of surgery, underlying disease (colon or rectal cancer), operative approach, extent of surgical resection, total operation time, preoperative SGOT. CHF (low event rate), platelets, and hematocrit (inconsistent assessment) not retained for regression model. ASA, American Society of Anesthesiologists; COPD, chronic obstructive pulmonary disease; ESPEN, European Society for Clinical Nutrition and Metabolism; LOS, length of stay; SSI, surgical site infection; OR, odds ratio.

(ASER): Referral to a dietician if BMI $<18.5 \mathrm{~kg} / \mathrm{m}^{2}(<20$ $\mathrm{kg} / \mathrm{m}^{2}$ if age $>65$ ) or unintentional weight loss of $>10 \%$ in last 6 months or eating less than $50 \%$ of a normal diet in the last week, and/or albumin $<3 \mathrm{~g} / \mathrm{dL}$ [6]. Furthermore, new Global Leadership Initiative in Malnutrition (GLIM) criteria, which were elaborated in dedicated consensus conferences in collaboration with different nutritional societies, have endorsed albumin $<3 \mathrm{~g} / \mathrm{dL}$ as a criterion of severe surgical risk, in line with the (updated) ESPEN guidelines for surgical patients $[20,24]$. The present study stresses the importance of albumin measurement as an important additional tool when nutritional screening is performed through clinical evaluation alone, espe- 
cially when screening is not performed by nutritional experts.

Arguably, preoperative albumin measurement comes with additional cost and systematic measurement within 21 days from surgery may represent a logistical challenge. However, severe hypoalbuminemia was associated with cost drivers prolonged LOS, unplanned readmission, and unplanned reoperation, in the present study and previous studies $[1,19,36]$. It has been shown that every dollar spent on nutritional therapy in hospitalized patients saves USD 52 of in-hospital costs [6,37]. Nevertheless, costeffectiveness of systematic albumin measurement needs to be further assessed. Adding albumin to conventional clinical screening may be most useful given the significant impact on postoperative outcomes if all analyzed criteria (BMI, weight loss, and hypoalbuminemia) were met (Fig. 3). Importantly, the benefits of preoperative nutritional therapy and prehabilitation have to be carefully balanced given a potential compromise of overall survival with delayed surgical management $[38,39]$.

The present results must be interpreted within the context of several limitations. First, patients with missing values were removed from our analysis, which may lead to selection bias. Furthermore, albumin levels may have been drawn based on clinical suspicion of malnutrition, potentially explaining the overrepresentation of the group with severe hypoalbuminemia. Second, the comparative baseline group with no severe malnutrition based on chosen criteria may have met other criteria for malnutrition that were not accounted for (Fig. 2). More specifically, ESPEN guidelines also endorse a wide range of measurements of body composition (i.e., lean body mass or muscle mass), which however were not available in the setting of this dataset. Moreover, details about screening modalities and institutional practice of participating centers were not available either. This needs to be considered when interpreting the results, especially regarding the baseline group, which may include patients fulfilling malnutrition criteria based on other definitions. Third, ACSNSQIP does not report cancer-specific outcomes, impeding investigation of long-term outcomes. Finally, albumin represents an unspecific marker for different disease states, which were excluded for this present analysis. However, albumin should not be considered a specific nutritional marker nor replace dedicated nutritional screening. In other words, this demonstrated that simplified nutritional screening through clinical parameters alone (particularly in a population with high average BMI) may lead to an underestimation of surgical risk. Hence, hypoalbuminemia as a surrogate of disease sever- ity could be a relevant additional tool in an "everyday" practice where systematic, comprehensive nutritional screening according to guidelines might be challenging.

In conclusion, this study revealed a strong association of severe hypoalbuminemia and postoperative morbidmortality in an unselected US cohort undergoing colorectal cancer surgery. Simplified clinical nutritional screening may lead to under-detection of patients at surgical risk. Further studies are needed to evaluate the cost-effectiveness of systematic serum albumin measurement.

\section{Statement of Ethics}

No ethical approval was needed as this study utilized a national database with no attempt to identify the patients.

\section{Conflict of Interest Statement}

There are no conflicts of interest to declare.

\section{Funding Sources}

This research did not receive any specific grant from funding agencies in the public, commercial, or non-profit sectors.

\section{Author Contributions}

Conception and design: F.G., M.A.A., W.P., K.T.B., A.D., K.L.M., and D.W.L. Acquisition of data: M.A.A. and F.G. Analysis and interpretation of data: M.A.A., F.G., W.P., K.T.B., A.D., K.L.M., and D.W.L. Drafting of the manuscript: F.G., M.A.A., W.P., K.T.B., A.D., K.L.M., and D.W.L. Critical revision of the manuscript: all authors. Final approval of the version to be published: all authors

\section{References}

1 Montomoli J, Erichsen R, Antonsen S, Nilsson T, Sørensen HT. Impact of preoperative serum albumin on 30-day mortality following surgery for colorectal cancer: a populationbased cohort study. BMJ Open Gastroenterol. 2015;2(1):e000047.

2 Lohsiriwat V, Lohsiriwat D, Boonnuch W, Chinswangwatanakul V, Akaraviputh T, Lert-Akayamanee N. Pre-operative hypoalbuminemia is a major risk factor for postoperative complications following rectal cancer surgery. World J Gastroenterol. 2008;14(8): 1248-51.

3 Lai CC, You JF, Yeh CY, Chen JS, Tang R, Wang JY, et al. Low preoperative serum albu$\mathrm{min}$ in colon cancer: a risk factor for poor outcome. Int J Colorectal Dis. 2011;26(4):473-81. 
4 Gibbs J, Cull W, Henderson W, Daley J, Hur K, Khuri SF. Preoperative serum albumin level as a predictor of operative mortality and morbidity: results from the National VA Surgical Risk Study. Arch Surg. 1999;134(1):3642.

5 Chandrasinghe PC, Ediriweera DS, Kumarage SK, Deen KI. Pre-operative hypoalbuminaemia predicts poor overall survival in rectal cancer: a retrospective cohort analysis. BMC Clin Pathol. 2013;13:12.

6 Wischmeyer PE, Carli F, Evans DC, Guilbert S, Kozar R, Pryor A, et al. American society for enhanced recovery and perioperative quality initiative joint consensus statement on nutrition screening and therapy within a surgical enhanced recovery pathway. Anesth Analg. 2018;126(6):1883-95.

7 McKenna N, Bews K, Al-Refaie W, Colibaseanu D, Pemberton J, Cima R, et al. Assessing malnutrition before major oncologic surgery: one size does not fit all. J Am Coll Surg. 2020; 230:451-60.

8 Budzynski J, Tojek K, Czerniak B, Banaszkiewicz Z. Scores of nutritional risk and parameters of nutritional status assessment as predictors of in-hospital mortality and readmissions in the general hospital population. Clin Nutr. 2016;35(6):1464-71.

9 Daniele A, Divella R, Abbate I, Casamassima A, Garrisi VM, Savino E, et al. Assessment of nutritional and inflammatory status to determine the prevalence of malnutrition in patients undergoing surgery for colorectal carcinoma. Anticancer Res. 2017;37(3):1281-7.

10 Cederholm T, Bosaeus I, Barazzoni R, Bauer J, Van Gossum A, Klek S, et al. Diagnostic criteria for malnutrition: an ESPEN consensus statement. Clin Nutr. 2015;34(3):335-40.

11 Zietarska M, Krawczyk-Lipiec J, Kraj L, Zaucha R, Malgorzewicz S. Nutritional status assessment in colorectal cancer patients qualified to systemic treatment. Contemp Oncol. 2017;21(2):157-61.

12 Karlsson S, Andersson L, Berglund B. Early assessment of nutritional status in patients scheduled for colorectal cancer surgery. Gastroenterol Nurs. 2009;32(4):265-70.

13 Meijers JM, van Bokhorst-de van der Schueren MA, Schols JM, Soeters PB, Halfens RJ. Defining malnutrition: mission or mission impossible? Nutrition. 2010;26(4):432-40.

14 Kondrup J, Rasmussen $\mathrm{HH}$, Hamberg $\mathrm{O}$, Stanga Z. Nutritional risk screening (NRS 2002): a new method based on an analysis of controlled clinical trials. Clin Nutr. 2003; 22(3):321-36.

15 Grass F, Cerantola Y, Schäfer M, Müller S, Demartines N, Hübner M. Perioperative nutrition is still a surgical orphan: results of a Swiss-Austrian survey. Eur J Clin Nutr. 2011; 65(5):642-7.

16 Ogden CL, Carroll MD, Fryar CD, Flegal KM. Prevalence of obesity among adults and youth: United States, 2011-2014. NCHS Data Brief. 2015;219:1-8.
17 Reber E, Gomes F, Vasiloglou MF, Schuetz P, Stanga Z. Nutritional risk screening and assessment. J Clin Med. 2019;8(7).

18 Almasaudi AS, Dolan RD, Edwards CA, McMillan DC. Hypoalbuminemia reflects nutritional risk, body composition and systemic inflammation and is independently associated with survival in patients with colorectal cancer. Cancers. 2020;12(7):1986.

19 Haskins IN, Baginsky M, Amdur RL, Agarwal S. Preoperative hypoalbuminemia is associated with worse outcomes in colon cancer patients. Clin Nutr. 2017;36(5):1333-8.

20 Weimann A, Braga M, Carli F, Higashiguchi T, Hübner M, Klek S, et al. ESPEN guideline: clinical nutrition in surgery. Clin Nutr. 2017; 36(3):623-50.

21 Weimann A, Braga M, Harsanyi L, Laviano A, Ljungqvist $\mathrm{O}$, Soeters $\mathrm{P}$, et al. ESPEN guidelines on enteral nutrition: surgery including organ transplantation. Clin Nutr. 2006;25(2): 224-44.

22 Levitt DG, Levitt MD. Human serum albumin homeostasis: a new look at the roles of synthesis, catabolism, renal and gastrointestinal excretion, and the clinical value of serum albumin measurements. Int J Gen Med. 2016;9: 229-55.

23 Raval MV, Pawlik TM. Practical guide to surgical data sets: national surgical quality improvement program (NSQIP) and pediatric NSQIP. JAMA Surg. 2018;153(8):764-5.

24 Cederholm T, Jensen GL, Correia MITD, Gonzalez MC, Fukushima R, Higashiguchi T, et al. GLIM criteria for the diagnosis of malnutrition: a consensus report from the global clinical nutrition community. Clin Nutr. 2019;38(1):1-9.

25 Ahima RS, Lazar MA. Physiology. The health risk of obesity: better metrics imperative. Science. 2013;341(6148):856-8.

26 Suarez-Llanos JP, Benitez-Brito N, VallejoTorres L, Delgado-Brito I, Rosat-Rodrigo A, Hernandez-Carballo C, et al. Clinical and cost-effectiveness analysis of early detection of patients at nutrition risk during their hospital stay through the new screening method CIPA: a study protocol. BMC Health Serv Res. 2017;17(1):292.

27 Bendersky V, Sun Z, Adam MA, Rushing C, Kim J, Youngwirth L, et al. Determining the optimal quantitative threshold for preoperative albumin level before elective colorectal surgery. J Gastrointest Surg. 2017;21(4):6929.

$28 \mathrm{Hu}$ WH, Chen HH, Lee KC, Liu L, Eisenstein S, Parry L, et al. Assessment of the addition of hypoalbuminemia to ACS-NSQIP surgical risk calculator in colorectal cancer. Medicine. 2016;95(10):e2999.
$29 \mathrm{Hu} \mathrm{WH}$, Eisenstein S, Parry L, Ramamoorthy S. Preoperative malnutrition with mild hypoalbuminemia associated with postoperative mortality and morbidity of colorectal cancer: a propensity score matching study. Nutr J. 2019;18(1):33.

30 Lobo DN, Gianotti L, Adiamah A, Barazzoni R, Deutz NEP, Dhatariya K, et al. Perioperative nutrition: recommendations from the ESPEN expert group. Clin Nutr. 2020;39: 3211-27.

31 Stratton RJ, Hackston A, Longmore D, Dixon R, Price S, Stroud M, et al. Malnutrition in hospital outpatients and inpatients: prevalence, concurrent validity and ease of use of the "malnutrition universal screening tool" ("MUST") for adults. Br J Nutr. 2004;92(5): 799-808.

32 Grass F, Hübner M, Schäfer M, Ballabeni P, Cerantola Y, Demartines N, et al. Preoperative nutritional screening by the specialist instead of the nutritional risk score might prevent excess nutrition: a multivariate analysis of nutritional risk factors. Nutr J. 2015;14:37.

33 Liu J, Wang F, Li S, Huang W, Jia Y, Wei C. The prognostic significance of preoperative serum albumin in urothelial carcinoma: a systematic review and meta-analysis. Biosci Rep. 2018;38(4)

34 Gu A, Malahias MA, Strigelli V, Nocon AA, Sculco TP, Sculco PK. Preoperative malnutrition negatively correlates with postoperative wound complications and infection after total joint arthroplasty: a systematic review and meta-analysis. J Arthroplasty. 2019;34(5): 1013-24.

35 Song GM, Tian X, Zhang L, Ou YX, Yi LJ, Shuai $\mathrm{T}$, et al. Immunonutrition support for patients undergoing surgery for gastrointestinal malignancy: preoperative, postoperative, or perioperative? A Bayesian network metaanalysis of randomized controlled trials. Medicine. 2015;94(29):e1225.

36 Bohl DD, Shen MR, Kayupov E, Della Valle CJ. Hypoalbuminemia independently predicts surgical site infection, pneumonia, length of stay, and readmission after total joint arthroplasty. J Arthroplasty. 2016;31(1): $15-21$.

37 Correia MI, Waitzberg DL. The impact of malnutrition on morbidity, mortality, length of hospital stay and costs evaluated through a multivariate model analysis. Clin Nutr. 2003; 22(3):235-9.

38 Grass F, Behm KT, Duchalais E, Crippa J, Spears GM, Harmsen WS, et al. Impact of delay to surgery on survival in stage I-III colon cancer. Eur J Surg Oncol. 2020;46(3):455-61.

39 Larson DW, Abd El Aziz MA, Mandrekar JN. How many lives will delay of colon cancer surgery cost during the COVID-19 pandemic? An analysis based on the US National Cancer Database. Mayo Clin Proc. 2020;95(8): $1805-7$. 